\title{
Manejo del dolor postoperatorio en cirugía por cáncer de mama
}

\author{
Postoperative pain management in breast cancer surgery
}

\author{
Oscar Abdiel Gómez Contreras, * Ludim Sarahí García Salazar* \\ Citar como: Gómez COA, García SLS. Manejo del dolor postoperatorio en cirugía por cáncer de \\ mama. Acta Med GA. 2022; 20 (1): 58-61. https://dx.doi.org/10.35366/103557
}

\section{Resumen}

El alivio del dolor es uno de los principales retos en nuestra labor y en los pacientes oncológicos este aspecto tiene especial relevancia por los efectos físicos y psicológicos que podría tener. El dolor agudo postoperatorio comúnmente existe después de una cirugía de cáncer de mama, se ha demostrado que 54\% de los pacientes que fueron sometidos a cirugía de cáncer de mama experimentaron dolor clínicamente importante, por lo que resulta fundamental comprender los factores de riesgo asociados a su presentación, así como las intervenciones que podemos realizar en el periodo perioperatorio para disminuir su prevalencia.

Palabras clave: Dolor, dolor postoperatorio, cirugía de mama, cáncer.

\section{INTRODUCCIÓN}

El alivio del dolor después de una cirugía continúa siendo uno de los principales retos médicos, el dolor postoperatorio manejado pobremente puede retrasar el alta hospitalaria y la recuperación. Recientes avances han ayudado a entender de mejor manera los mecanismos del dolor, así como su fisiología y farmacología, se han establecido guías e introducido dispositivos para su manejo, sin embargo, el subtratamiento del dolor postoperatorio continúa siendo un problema a nivel mundial. ${ }^{1,2}$

\footnotetext{
* Departamento de Anestesiología del Hospital General de Zona No. 11, IMSS. Nuevo Laredo, Tamaulipas.
}

Correspondencia:

Dr. Oscar Abdiel Gómez Contreras

Correo electrónico: abdiel_gomezc@hotmail.com

Aceptado: 31-05-2021.

www.medigraphic.com/actamedica

\section{Abstract}

Pain relief is one of the main challenges of our work and in cancer patients, this aspect takes on special relevance due to the physical and psychological effects it can have. Acute postoperative pain commonly exists after breast cancer surgery, it has been shown that $54 \%$ of patients who had surgery for breast cancer experienced clinically important pain, so it is important to understand the risk factors associated with its presentation, as well as the interventions that we can carry out in the perioperative period to reduce its prevalence.

Keywords: Pain, postoperative pain, breast surgery, cancer.

El dolor agudo postoperatorio comúnmente existe después de una cirugía de cáncer de mama. Katz y colaboradores demostraron que $54 \%$ de los pacientes que fueron sometidos a cirugía de cáncer de mama experimentaron dolor clínicamente importante. ${ }^{3}$ El dolor postoperatorio puede ser afectado por el tipo de procedimiento quirúrgico efectuado, siendo más severo y prolongado en cirugías que involucren la reconstrucción de la mama. ${ }^{4,5}$ Las sensaciones anormales son menos frecuentes en cirugías que involucran biopsia de ganglio centinela en comparación con aquellas a nivel axilar. ${ }^{6}$

\section{Factores de riesgo asociados con dolor agudo postoperatorio en cirugía de cáncer de mama}

Los factores asociados con dolor agudo postoperatorio son, hasta cierto punto, conocidos. Una revisión ${ }^{7}$ sugiere que cuatro factores se asocian significativamente a la intensidad del dolor postoperatorio: dolor preoperatorio, ansiedad, edad, tipo de cirugía.

Dolor preoperatorio. El dolor preoperatorio en mama y axila en pacientes que serán sometidos a cirugía de cáncer 
de mama tiene un efecto significativo en el consumo de medicación analgésica en el postoperatorio.

Un estudio reciente mostró que hasta una cuarta parte de los pacientes que serán sometidos a este tipo de procedimientos tienen dolor en la mama o axila y se sugiere que los genes que regulan las citosinas pro- $y$ antiinflamatorias tienen un papel importante para su desarrollo. ${ }^{8}$

Las causas potenciales para el desarrollo de dolor en pacientes con cáncer de mama antes de ser sometidas a cirugía incluyen la liberación de mediadores alogénicos desde el tumor, ${ }^{9}$ daño perineural por el cáncer e inflamación asociada a lesión tisular después de una biopsia de mama. Esta lesión tisular se asocia con liberación de citosinas proinflamatorias como IL-1, TNF- $\alpha$, que resulta en dolor de tipo inflamatorio. Además, variaciones en genes que involucran vías inflamatorias se asocian con incremento en dolor agudo y por cáncer. ${ }^{10}$

Edad. Una edad más joven ha sido consistentemente encontrada como factor de riesgo para desarrollar dolor después de ser sometido a cirugía de cáncer de mama, tanto en su forma aguda como crónica. ${ }^{7,11-13}$

Se han estudiado distintas causas para explicar por qué las mujeres más jóvenes reportan más dolor, dentro de las que se incluyen un tumor más grande, peor grado histológico, receptores tumorales negativos a estrógenos, ${ }^{14}$ disminución de la percepción del dolor relacionado con la edad, ${ }^{15}$ diferencias en cómo los grupos de edad reportan el dolor, así como diferentes mecanismos psicológicos. ${ }^{16}$

Tipo de cirugía. La disección de nódulos linfáticos axilares ha sido consistentemente mostrada como factor de riesgo para el desarrollo de dolor postoperatorio más severo, así como alteraciones en la sensibilidad en comparación con la biopsia de ganglio centinela. De hecho, la disección de nódulos linfáticos axilares, en comparación con la biopsia de ganglio centinela, es uno de los factores de riesgo mayores para el desarrollo de linfedema del brazo. Asimismo, el daño del nervio intercostobraquial ha sido propuesto como uno de los mecanismos principales del dolor después de la cirugía de cáncer de mama. ${ }^{17-19}$

Ansiedad. Los altos niveles de ansiedad preoperatoria y distrés psicológico son conocidos por elevar la intensidad del dolor postoperatorio, esto se ha demostrado en múltiples estudios. ${ }^{20,21}$

De igual forma la expectativa sobre el dolor puede afectar la respuesta psicológica y fisiológica, ${ }^{22}$ afectando principalmente la percepción de la intensidad del dolor postoperatorio.

\section{Importancia de la predicción del dolor agudo postoperatorio}

Como ya se comentó previamente, el adecuado manejo del dolor agudo postoperatorio per se representa parte im- portante del tratamiento, no sólo de pacientes con cáncer de mama, sino de cualquier procedimiento quirúrgico, ya que de no realizarse adecuadamente presenta efectos a nivel biopsicosocial.

Al hablar propiamente de la cirugía de cáncer de mama, el dolor agudo postoperatorio se considera un factor de riesgo para desarrollar dolor crónico, por lo que es importante determinar su relación con características demográficas, clínicas y psicológicas. Además, la identificación de factores asociados con dolor agudo postoperatorio pueden ayudar a determinar los mecanismos fisiopatológicos y psicológicos para desarrollar dolor crónico postoperatorio. ${ }^{23}$

Existe un test, el Pain Sensitivity Questionnaire (PSQ), que se ha utilizado para predecir dolor postoperatorio basándose en pruebas que permiten evaluar el dolor preoperatorio; sin embargo, existe un solo estudio que evalúa su sensibilidad en pacientes postoperadas de mastectomía que presenta algunas limitaciones, como el número de pacientes evaluados, por lo que se considera no concluyente. ${ }^{24,25}$

\section{Manejo del dolor agudo postoperatorio en pacientes sometidas a cirugía por cáncer de mama}

Terapia analgésica preventiva. El uso de este tipo de terapia en estos procedimientos está enfocado principalmente al manejo multimodal para la prevención del dolor crónico con limitados efectos en el dolor agudo.

Es conocido que la incisión quirúrgica induce hiperalgesia, que puede contribuir a dolor persistente después de la cirugía, asimismo, existe evidencia de que un adecuado manejo del dolor agudo postoperatorio ayuda a disminuir el riesgo de desarrollo de dolor crónico. En años recientes, la pregabalina se ha introducido como adyuvante en el manejo de dolor postoperatorio. ${ }^{26}$

Un gran número de ensayos clínicos han evaluado la eficacia y efectos adversos de la pregabalina en la reducción del dolor agudo postoperatorio; sin embargo, estos estudios han mostrado resultados inconsistentes por la diferencia en las dosis utilizadas, el régimen de dosificación, la severidad y el tipo de dolor. Algunos estudios han reportado ningún efecto analgésico con el uso de pregabalina perioperatoria ${ }^{27}$ y otros prueban una reducción en el consumo de analgésicos y en las escalas de dolor. ${ }^{28}$

Bloqueos regionales. Alternativas a la anestesia general como la medicación preoperatoria, analgesia neuropática y anestesia local o regional se han explorado para reducir las complicaciones propias de la anestesia general, así como para un mejor control del dolor. La infiltración regional de agentes anestésicos o toxina botulínica resulta en adecuada 
analgesia, disminuyendo la morbilidad perioperatoria y los días de estancia hospitalaria. ${ }^{29-32}$

\section{Bloqueo torácico paravertebral}

Particularmente, el bloqueo torácico paravertebral es considerado una opción viable que ofrece beneficios, mejorando la anestesia quirúrgica y la analgesia postoperatoria. Esta técnica se ha asociado con una recuperación más rápida y menos dolor postoperatorio, así como menores requerimientos de analgésicos postoperatorios en comparación con la anestesia general. ${ }^{33}$

Existe un estudio que compara la influencia del tipo de anestesia en la regulación inmunológica con el uso del bloqueo torácico paravertebral junto con propofol contra la anestesia general basada en sevoflurano más opioide, se observa que en el grupo de los pacientes en los que se utilizó bloqueo torácico paravertebral más propofol hubo un incremento significativo en los niveles de IL-10, que inhibe la producción de citosinas proinflamatorias y promueve la actividad antitumoral y antimetastásica, aunque su papel en el cáncer de mama no está claro aún. ${ }^{34}$

\section{Bloqueo del nervio pectoral}

Es un procedimiento relativamente sencillo, y que se puede realizar antes de la cirugía, que bloquea los nervios pectorales lateral y medial, ramas del plexo braquial y responsables de la inervación motora de los músculos pectoral mayor y menor. ${ }^{35}$ El bloqueo del nervio pectoral provee analgesia a la parte interna de la mama, a las ramas intercostobraquial y cutánea lateral de los nervios intercostales, al nervio cutáneo medial del brazo y a los nervios torácico largo y toracodorsal. ${ }^{36}$

\section{Bloqueo del plano transverso torácico}

El bloqueo del plano transverso guiado por ultrasonido puede bloquear múltiples ramas anteriores de los nervios intercostales que dominan la región mamaria interna. ${ }^{37}$ Sin embargo, se ha demostrado que su combinación junto con el bloqueo del nervio pectoral provee mejor analgesia que los dos métodos por sí solos en cirugía de cáncer de mama. ${ }^{38}$

Analgesia sistémica. Muchos estudios han mostrado que la analgesia regional provee una recuperación más funcional y un control del dolor superior después de la cirugía de cáncer de mama. No obstante, la analgesia regional para cirugía de cáncer de mama no es enteramente utilizada por los riesgos propios o las dificultades técnicas que pudiera presentar (lesión nerviosa, sangrado en pacientes anticoagulados, etcétera). ${ }^{39}$
De manera reciente, se han utilizado lidocaína y magnesio perioperatorios, mostrando una disminución del dolor postoperatorio y reducción en el consumo de analgésicos. Sin embargo, hay un solo estudio que compara ambos fármacos en el periodo perioperatorio en cirugía de cáncer de mama y que muestra superioridad de la lidocaína, ya que el sulfato de magnesio sólo es efectivo para reducir el consumo de opioides intraoperatorios y para una disminución del dolor en las primeras horas de la cirugía, mientras que la lidocaína, además, reduce la intensidad del dolor crónico postoperatorio. ${ }^{40}$

\section{CONCLUSIÓN}

El manejo del dolor postoperatorio debe ser integral, desde la detección para su presentación en la evaluación preanestésica hasta el periodo perioperatorio, escogiendo las mejores medidas farmacológicas y no farmacológicas que puedan impactar de forma positiva en nuestro paciente, la elección de ésta será según distintos factores y la expertis de quien implementará las técnicas, pero siempre basados en la mejor evidencia posible.

En esta revisión se busca dar al lector un panorama amplio del manejo del dolor postoperatorio en cirugía por cáncer de mama, así como las técnicas que se pueden emplear tanto para su prevención como para el tratamiento agudo.

\section{REFERENCIAS}

1. Wu CL, Raja SN. Treatment of acute postoperative pain. Lancet. 2011; 377 (9784): 2215-2225.

2. Werner MU, Soholm L, Rotboll-Nielsen P, Kehlet H. Does an acute pain service improve postoperative outcome? Anesth Analg. 2002; 95 (5): 1361-1372.

3. Katz J, Poleshuck EL, Andrus CH, Hogan LA, Jung BF, Kulick DI et al. Risk factors for acute pain and its persistence following breast cancer surgery. Pain. 2005; 119 (1-3): 16-25.

4. Wallace MS, Wallace AM, Lee J, Dobke MK. Pain after breast surgery: a survey of 282 women. Pain. 1996; 66 (2-3): 195-205.

5. Legeby M, Segerdahl M, Sandelin K, Wickman M, Ostman K, Olofsson $\mathrm{Ch}$. Immediate reconstruction in breast cancer surgery requires intensive post-operative pain treatment but the effects of axillary dissection may be more predictive of chronic pain. Breast. 2002; 11 (2): 156-162.

6. Baron RH, Fey JV, Borgen PI, Stempel MM, Hardick KR, Van Zee KJ. Eighteen sensations after breast cancer surgery: a 5-year comparison of sentinel lymph node biopsy and axillary lymph node dissection. Ann Surg Oncol. 2007; 14 (5) :1653-1661.

. Ip HY, Abrishami A, Peng PW, Wong J, Chung F. Predictors of postoperative pain and analgesic consumption: a qualitative systematic review. Anesthesiology. 2009; 111 (3): 657-677.

8. McCann B, Miaskowski C, Koetters T, Baggott C, West C, Levine JD et al. Associations between pro- and anti-inflammatory cytokine genes and breast pain in women prior to breast cancer surgery. J Pain. 2012; 13 (5): 425-437.

9. Grivennikov SI, Karin M. Inflammatory cytokines in cancer: tumour necrosis factor and interleukin 6 take the stage. Ann Rheum Dis. 2011; 70 Suppl 1: i104-i108. 
10. Bessler H, Shavit Y, Mayburd E, Smirnov G, Beilin B. Postoperative pain, morphine consumption, and genetic polymorphism of IL-1 beta and IL-1 receptor antagonist. Neurosci Lett. 2006; 404 (1-2): 154-158.

11. Andersen KG, Kehlet $\mathrm{H}$. Persistent pain after breast cancer treatment: a critical review of risk factors and strategies for prevention. J Pain. 2011; 12 (7): 725-746.

12. Kehlet $\mathrm{H}$, Jensen TS, Woolf CJ. Persistent postsurgical pain: risk factors and prevention. Lancet. 2006; 367 (9522): 1618-1625.

13. Jung BF, Ahrendt GM, Oaklander AL, Dworkin RH. Neuropathic pain following breast cancer surgery: proposed classification and research update. Pain. 2003; 104 (1-2): 1-13.

14. Kroman N, Jensen MB, Wohlfahrt J, Mouridsen HT, Andersen PK, Melbye $M$. Factors influencing the effect of age on prognosis in breast cancer: population based study. BMJ. 2000; 320 (7233): 474-478.

15. Gagliese L, Katz J. Age differences in postoperative pain are scale dependent: a comparison of measures of pain intensity and quality in younger and older surgical patients. Pain. 2003; 103 (1-2): 11-20.

16. Andersen KG, Jensen MB, Tvedskov TF, Kehlet H, Gartner R, Kroman $\mathrm{N}$. Persistent pain, sensory disturbances and functional impairment after immediate or delayed axillary lymph node dissection. Eur J Surg Oncol. 2013; 39(1): 31-35.

17. Verma S, Kala S, Bhargava R, Yadav G, Singh RK, Maurya RK et al. Evaluation of the role of preservation of the intercostobrachial nerve on the post-mastectomy pain syndrome in breast cancer patients of North India. Int J Surg. 2010; 23 (2): 1-17.

18. Mejdahl MK, Andersen KG, Gartner R, Kroman N, Kehlet H. Persistent pain and sensory disturbances after treatment for breast cancer: six year nationwide follow-up study. BMJ. 2013; 346: f1865.

19. Burns JW, Moric M. Psychosocial factors appear to predict postoperative pain: Interesting, but how can such information be used to reduce risk? Tech Reg Anesth Pain Manag. 2011; 15: 90-99.

20. Montgomery GH, Schnur JB, Erblich J, Diefenbach MA, Bovbjerg DH. Presurgery psychological factors predict pain, nausea, and fatigue one week after breast cancer surgery. J Pain Symptom Manage. 2010; 39 (6): 1043-1052.

21. Logan DE, Rose JB. Is postoperative pain a self-fulfilling prophecy? Expectancy effects on postoperative pain and patient-controlled analgesia use among adolescent surgical patients. J Pediatr Psychol. 2005; 30 (2): 187-196.

22. Kirsch I. Response expectancy as a determinant of experience and behavior. Am Psychol. 1985; 40 (11): 1189-1202.

23. Tracey I. Getting the pain you expect: mechanisms of placebo, nocebo and reappraisal effects in humans. Nat Med. 2010; 16 (11): 1277-1283.

24. Ozalp G, Sarioglu R, Tuncel G, Aslan K, Kadiogullari N. Preoperative emotional states in patients with breast cancer and postoperative pain. Acta Anaesthesiol Scand. 2003; 47 (1): 26-29.

25. Rehberg B, Mathivon S, Combescure C, Mercier Y, Savoldelli GL. Prediction of acute postoperative pain following breast cancer surgery using the pain sensitivity questionnaire: a cohort study. Clin J Pain. 2017; 33 (1): 57-66.

26. Ruscheweyh R, Marziniak M, Stumpenhorst F, Reinholz J, Knecht S. Pain sensitivity can be assessed by self-rating: Development and validation of the Pain Sensitivity Questionnaire. Pain. 2009; 146 (12): $65-74$.
27. Gilron I. Gabapentin and pregabalin for chronic neuropathic and early postsurgical pain: current evidence and future directions. Curr Opin Anaesthesiol. 2007; 20 (5): 456-472.

28. Chang SH, Lee HW, Kim HK, Kim SH, Kim DK. An evaluation of perioperative pregabalin for prevention and attenuation of postoperative shoulder pain after laparoscopic cholecystectomy. Anesth Analg. 2009; 109 (4): 1284-1286.

29. Boughey JC, Goravanchi F, Parris RN, Kee SS, Frenzel JC, Hunt KK et al. Improved postoperative pain control using thoracic paravertebral block for breast operations. Breast J. 2009; 15 (5): 483-488.

30. Aufforth R, Jain J, Morreale J, Baumgarten R, Falk J, Wesen C. Paravertebral blocks in breast cancer surgery: is there a difference in postoperative pain, nausea, and vomiting? Ann Surg Oncol. 2012; 19 (2): 548-552.

31. Bauer M, George JE 3rd, Seif J, Farag E. Recent advances in epidural analgesia. Anesthesiol Res Pract. 2012; 2012: 309219.

32. Bhuvaneswari V, Wig J, Mathew PJ, Singh G. Post-operative pain and analgesic requirements after paravertebral block for mastectomy: A randomized controlled trial of different concentrations of bupivacaine and fentanyl. Indian J Anaesth. 2012; 56 (1): 34-39.

33. Pusch F, Freitag H, Weinstabl C, Obwegeser R, Huber E, Wildling E. Single-injection paravertebral block compared to general anaesthesia in breast surgery. Acta Anaesthesiol Scand. 1999; 43 (7): 770-774.

34. Deegan CA, Murray D, Doran P, Moriarty DC, Sessler DI, Mascha $E$ et $\mathrm{al}$. Anesthetic technique and the cytokine and matrix metalloproteinase response to primary breast cancer surgery. Reg Anesth Pain Med. 2010; 35 (6): 490-495.

35. Blanco R, Fajardo M, Parras Maldonado T. Ultrasound description of Pecs II (modified Pecs I): a novel approach to breast surgery. Rev Esp Anestesiol Reanim. 2012; 59 (9): 470-475.

36. Blanco R. The 'pecs block': a novel technique for providing analgesia after breast surgery. Anaesthesia. 2011; 66 (9): 847-848.

37. Ueshima H, Kitamura A. Blocking of multiple anterior branches of intercostal nerves (Th2-6) using a transversus thoracic muscle plane block. Reg Anesth Pain Med. 2015; 40 (4): 388.

38. Ueshima $\mathrm{H}$, Otake $\mathrm{H}$. Addition of transversus thoracic muscle plane block to pectoral nerves block provides more effective perioperative pain relief than pectoral nerves block alone for breast cancer surgery. Br J Anaesth. 2017; 118 (3): 439-443.

39. Vigneault L, Turgeon AF, Coté D, Lauzier F, Zarychanski R, Moore L et al. Perioperative intravenous lidocaine infusion for postoperative pain control: a meta-analysis of randomized controlled trials. Can J Anaesth. 2011; 58 (1): 22-37.

40. Kim MH, Lee KY, Park S, Kim SI, Park HS, Yoo YC. Effects of systemic lidocaine versus magnesium administration on postoperative functional recovery and chronic pain in patients undergoing breast cancer surgery: A prospective, randomized, double-blind, comparative clinical trial. PLoS One. 2017; 12 (3): e0173026.

Conflicto de intereses: Oscar Abdiel Gómez Contreras y Ludim Sarahí García Salazar declaran que no tienen conflicto de intereses.

Este artículo no contiene ningún estudio con sujetos humanos o animales realizado por ninguno de los autores. 